\title{
Left sided omental torsion with left inguinal hernia: a rare entity
}

\author{
Ranjana Gupta $\cdot$ Puneet Mittal • Gaurav Mittal
}

Published online: 1 October 2011

(C) Indian Society of Gastroenterology 2011

A 29-year-old man presented with history of acute pain in left upper quadrant and lumbar region since 2 days. There was no history of fever or vomiting. Patient had a left-sided inguinal hernia since 5 years.

CT scan revealed a fatty mass in left side of omentum. A central hyperdense structure with surrounding whirling streaks was seen (Fig. 1). There was marked stranding of

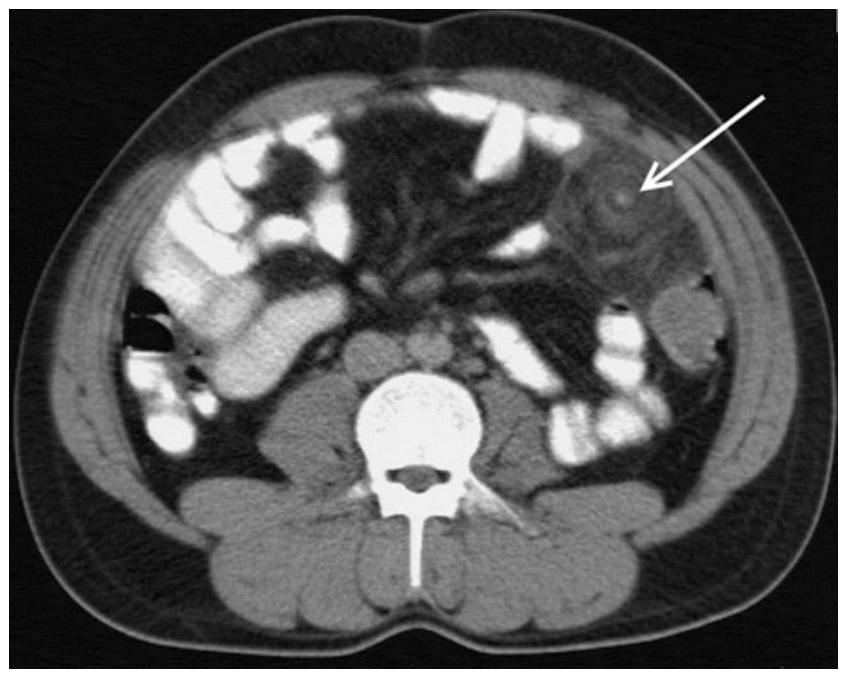

Fig. 1 Axial CT sections show fatty mass with central hyperdense structure with concentric whorled appearance

\section{R. Gupta}

Department of Radiodiagnosis,

Dayanand Medical College and Hospital,

Ludhiana, Punjab 1410 01, India

P. Mittal $(\bowtie)$

Department of Radiodiagnosis,

Punjab Institute of Medical Sciences,

Jalandhar, Punjab 144 006, India

e-mail: drpuneetmittal@gmail.com

\section{G. Mittal}

Department of Medicine, Punjab Institute of Medical Sciences, Jalandhar, Punjab 144 006, India

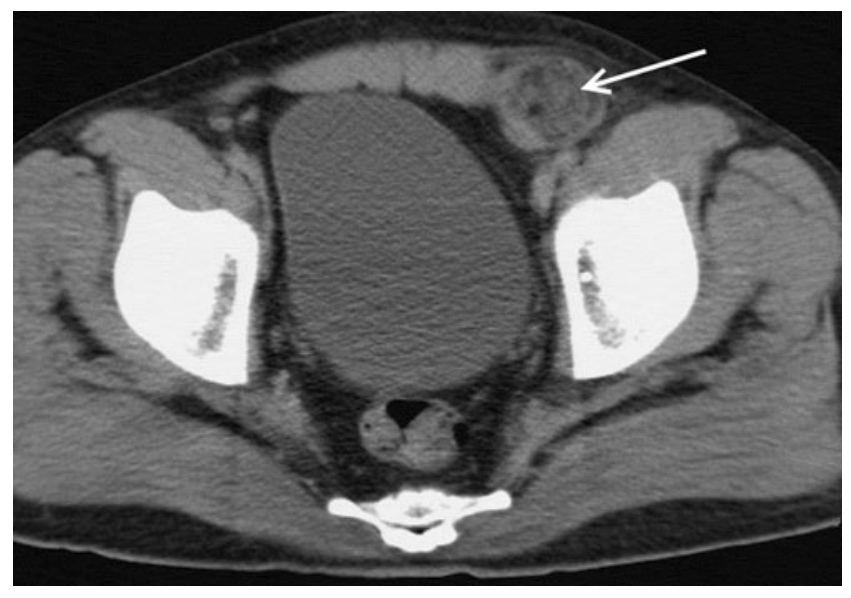

Fig. 2 Axial CT sections show extension of the fatty mass into left inguinal canal

the omentum and the stranding continued inferiorly into left inguinal hernia sac (Fig. 2). The diagnosis of omental torsion with hernia was confirmed on laparotomy.

Omental torsion usually occurs on the right side because of greater mobility of the right omentum. A left-sided omental torsion is rare and is difficult to diagnose clinically. Torsion may be primary, or secondary to underlying causes like hernia, tumors or adhesions [1]. Omental torsion results in vascular congestion with subsequent obstruction and omental infarction.

Omental torsion is only rarely diagnosed preoperatively. $\mathrm{CT}$ is the investigation of choice for making the diagnosis. Omental torsion presents with characteristic fatty mass with central hyperdensity with surrounding whirling pattern [2].

\section{References}

1. Wong EMF, Ka SYJ, Chau WK, Tsui P. Left-sided torsion of the greater omentum. J Hong Kong Col Radiol. 2010;13:154-7.

2. Hirano Y, Oyama K, Nozawa H, et al. Left-sided omental torsion with inguinal hernia. World J Gastroenterol. 2006;12:662-4. 